\title{
ESTRATEGIAS DE MARKETING SOCIAL PARA PREVENIR LOS RIESGOS PSICOSOCIALES EN LAS PYMES DE GUAYAQUIL
}

\author{
Ing. Yuryko Yamileth Sánchez Ruíz \\ Estudiante. Universidad Laica Vicente Rocafuerte de Guayaquil \\ https://orcid.org/0000-0003-2785-6493 \\ ysanchezr@ulvr.edu.ec \\ Ing. Mario David Vera Sellán \\ Estudiante. Universidad Laica Vicente Rocafuerte de Guayaquil \\ https://orcid.org/0000-0003-3978-6744 \\ mveras@ulvr.edu.ec
}

Mgtr. Marisol Jacqueline Idrovo Avecillas

Docente. Universidad Laica Vicente Rocafuerte de Guayaquil https://orcid.org/0000-0002-0176-9917 midrovoa@ulvr.edu.ec

Para citar este artículo puede utilizar el siguiente formato:

Yuryko Yamileth Sánchez Ruíz, Mario David Vera Sellán y Marisol Jacqueline Idrovo Avecillas:

"Estrategias de marketing social para prevenir los riesgos psicosociales en las PYMES de Guayaquil", Revista Observatorio de la Economía Latinomericana (Vol 19, № 5, pp. 45-59, julio-septiembre 2021). En línea:

https://doi.org/10.51896/oel/OZKY1437

\section{RESUMEN}

La presente investigación tuvo como finalidad identificar las condiciones laborales en que se encuentran las PYMES de la ciudad de Guayaquil para gestionar apropiadamente los riesgos psicosociales y prevenirlos con la ayuda del Marketing Social. La metodología de investigación aplicada se llevó a cabo a través de un enfoque mixto, utilizando los métodos deductivo e inductivo; mediante el método deductivo se tomó una población general, que se llevó a la muestra idéntica y por medio del método inductivo se interpretó discernimientos que fueron llevados a generalidades. La investigación se centra en las PYMES dedicadas a la Actividad de Comercio - Grupo G, ya que pertenecen a la mayor parte de empresas que son afectadas y se encuentran domiciliadas en la ciudad de Guayaquil, por lo que se consideró a empleadores, representantes de trabajadores y expertos en seguridad y salud ocupacional con la finalidad de obtener información permita brindar solución a la problemática identificada. Entre los principales hallazgos extraídos de la investigación se determinó que de la muestra el 58,37\% de los empleadores de las PYMES no cumplen con los programas de riesgos psicosociales en su mayoría por desconocimiento y porque no lo consideran necesario por lo que es necesario e indispensable realizar 
estrategias de marketing social a través de una campaña social a empleadores para brindar el conocimiento sobre los riesgos psicosociales y al ser concientizados se mitiguen los riesgos y se mejore la calidad de vida de los miembros de la empresa, mejorando la productividad y competitividad en la empresa.

Palabras clave:

Prevención de riesgos, Conciencia, Salud, Trabajador, Organización.

\section{SOCIAL MARKETING STRATEGIES TO PREVENT PSYCHOSOCIAL RISKS IN SMEs IN GUAYAQUIL}

\section{ABSTRACT}

The present investigation was to purpose identify the labor conditions in which SMEs in the city of Guayaquil meet in order to properly manage psychosocial risks and prevent them with the assistance of Social Marketing. The applied research methodology was carried out through a mixed approach, using deductive and inductive methods; by means of the deductive method a general population was taken, which was taken to the identical sample and by means of the inductive method was interpreted discernments that were taken to generalities. The investigation focuses on SMEs dedicated to the Business Activity - Group G, which belong to the majority of companies that are affected and are domiciled in the city of Guayaquil, for which employers, representatives of workers were considered. and experts in security and occupational health with the purpose of obtaining information allow to provide a solution to the identified problem. Among the main factors extracted from the investigation, it was determined that $58.37 \%$ of SMEs employers do not comply with psychosocial risk programs in their majority for ignorance and because they do not consider it necessary for what is necessary. It is essential to carry out social marketing strategies through a social campaign for employers to provide knowledge about psychosocial risks and to be made aware if they mitigate risks and improve the quality of life of members of the company, improving productivity and competitiveness the company.

Keywords: Risk prevention, Awareness, Health, Worker, Organization.

\section{INTRODUCCIÓN}

Los riesgos psicosociales inciden en las pymes tanto en colaboradores y empleadores, su procedencia está relacionada con el incumplimiento de las normativas vigentes del Ministerio de Trabajo y los artículos reflejados en el Código de Trabajo, el incumplimiento de la ley y de las normativas repercuten en sanciones económicas a los empleadores, afectando el desempeño laboral de los trabajadores por lo que desencadena una baja calidad de los productos y/o servicios que se comercializan causando pérdidas en la empresa, todos estos factores que se mencionan logra que incremente la tensión y los trastornos mentales, "de acuerdo a lo reportado por la OIT en Finlandia, más del $50 \%$ de los trabajadores sufren algún tipo de síntomas relacionados con el estrés, tales como ansiedad, sentimientos depresivos, dolor físico, exclusión social y trastornos del sueño" (Ministerio de 
Salud Pública del Ecuador, 2019, p. 62).

En las estadísticas del Seguro de Riesgos del Trabajo SGRT en el 2020 reportaron 39.653 atenciones médicas, el cual comprende a 1.669 atenciones por enfermedades profesionales y 37.984 por accidentes del trabajo en Ecuador, sin embargo, en la provincia del Guayas fue una de las provincias que tuvo más atenciones el cual reflejó 13.596 atenciones por accidente y/o enfermedades en el trabajo (SGRT, 2020). Si los riesgos psicosociales no son atendidos de forma apropiada, las cifras de atenciones médicas y/o enfermedades en el trabajo no lograrán reducirse, ya que lamentablemente son cifras que van incrementando con el pasar del tiempo.

"Durante los últimos meses que el país está enfrentando la pandemia global del Covid-19, se ha percibido ciertas características emocionales en los trabajadores de la empresa, que pueden significar o dar origen a algún tipo de riesgo psicosocial" (Robalino Zumba, 2020, p. 3). Las condiciones laborales en una emergencia demandan un elevado esfuerzo mental, emocional y conductual, generando como resultado el estrés adaptativo que, si se mantiene de manera prolongada, podría convertirse en distrés 'estrés negativo' el cual se manifiesta como respuesta al esfuerzo excesivo, provocando afecciones cognitivas, emocionales, conductuales y física (Ministerio de Salud Pública del Ecuador, 2020).

Las condiciones de trabajo, entendidas como las características del medio ambiente físico y organizativo en que se desarrollan las tareas, así como las condiciones de empleo, referidas a la naturaleza de la relación entre empleador y el trabajador, generan un impacto significativo en la salud de las personas. (Ministerio de Salud Pública del Ecuador, 2019, p. 36)

Las empresas grandes y las PYMES deben considerar una actitud preventiva ante las circunstancias que se presenten dentro de la organización al momento de ejecutar sus actividades laborales, por ende, una empresa que logra reconocer sus falencias a tiempo logrará que los trabajadores gocen de buena salud y seguridad dentro de la misma. Sandra Tandalla, en su tesis para obtener el título de Máster Universitario en Seguridad y Salud en el Trabajo: Prevención de Riesgos Laborales, con el tema, "Cultura Preventiva. Estrategias de Cambio Cultural de obligatoriedad a concienciación de prevención de riesgos laborales para generar excelencia, salud empresarial y sostenibilidad." concluye que: "la gestión de prevención de riesgos laborales se debe empezar con la consulta a los trabajadores, saber las necesidades, sus percepciones; y por lo tanto vincular a todos los niveles jerárquicos basándose en las condiciones de trabajo y adecuado liderazgo" (Tandalla, 2018, p. 38).

Por lo que deben ser atendidos prioritariamente. Las empresas grandes y las PYMES deben considerar una actitud preventiva ante las circunstancias que se presenten dentro de la organización al momento de ejecutar sus actividades laborales, por ende, una empresa que logra reconocer sus falencias a tiempo logrará que los trabajadores gocen de buena salud y seguridad dentro de la misma. La herramienta de Marketing Social se considera que puede ser empleada en el trabajo social; es decir, 
para emplear Marketing Social es necesario investigar y analizar todos los comportamientos, deseos, percepciones y actitudes para que al promover las estrategias de Marketing Social ocasionen un mayor impacto.

\section{Marketing Social}

El Marketing Social, es una disciplina de Marketing, orientada hacia el bienestar social, que está encaminado a los valores de los seres humanos para detectar y satisfacer necesidades de tipo social, humanitarias y/o espirituales. En el libro de (Kotler \& Armstrong, 2013) indica que según el Social Marketing Institute (SMI) define al Marketing Social como "el uso de conceptos y herramientas de marketing comercial dentro de programas diseñados para influir en el comportamiento de los individuos a fin de mejorar su bienestar y el de la sociedad" (p. 201).

El Marketing Social se apoya de las herramientas del marketing comercial para influir en el comportamiento de los seres humanos, mediante estrategias que logren llegar al público objetivo deseado. Esta actividad se realiza por cualquier tipo de institución, por lo general el sector gubernamental es el que ha implementado con mayor frecuencia esta herramienta; aunque cabe mencionar, que en el transcurso del tiempo sus líneas de acción se han ido transformando para ejecutar campañas que promueven el cambio a los problemas más comunes que existen en la sociedad.

\section{Las Estrategias Integradas de Marketing Social}

Las estrategias del Marketing Social se generan de manera natural a partir del análisis exhaustivo de la población objetivo o mercado meta, de la competencia, de los proveedores, etc. Por lo que se mencionan algunas estrategias derivadas de ese análisis:

\section{Mezcla de Marketing Social}

La aplicación de las estrategias de la mezcla de marketing social o marketing mix es una ventaja competitiva que toda organización tiene posibilidad de elaborar en función de sus recursos, debilidades y visión estratégica, para de esa forma atender las necesidades de la población o público objetivo, ya que de esa forma se satisfacen las carencias detectadas y se incrementan los índices de productividad y rentabilidad en las empresas. La mezcla del marketing dependerá de la organización que será responsable del diseño del producto o servicio, fijación de precio, empleo de las herramientas de promoción, la ubicación, el personal y de la presentación de la imagen. Implementar la mezcla de Marketing social también es esencial para integrar las estrategias de forma organizada abarcando aspectos importantes que brindarán ese beneficio bilateral que tiene la entrega de los productos y/o servicios al público objetivo.

\section{Estrategias de Cambio}

Según Kotler y Roberto, destacan cuatro estrategias principales de cambio que ayuda al marketing social en cumplir con su objetivo de brindar un bienestar social, para lo cual se utilizará como ejemplo una campaña de lucha contra el tabaquismo (Seguí \& Pérez, 2016): 
- Tecnológicas: Hace referencia a la tecnología de modificación del producto, tecnología de sustitución del producto, tecnología de innovación del producto.

- Económicas: Estas tratan de imponer costos sobre la conducta no deseable y de recompensar la conducta deseable. Por ejemplo. Adicionar un impuesto elevado a la caja de cigarrillos.

- Político-legal: Se pueden aprobar leyes que restrinjan actividades que permitan menorar el consumo de cigarrillo en este caso.

- Educativa: En esta estrategia puede intervenir el Estado y asociaciones sin fines de lucro.

\section{Marketing Social en la Salud}

La aplicación de Marketing Social en el campo de la promoción de la Salud, se apoya en las intervenciones educativas para la salud en cuanto al manejo de riesgos personales, en el desarrollo de aptitudes, capacidades y competencias de salud, basándose en promover (la conducta saludable), la comunidad (audiencia o grupo objetivo) y diferentes variables (individuales, colectivas, ambientales y culturales), con el fin de reforzar hábitos y comportamientos saludables.

El Marketing Social en la Salud implementa el uso de estrategias y medios de comunicación como:

- Comunicación Educativa en Salud: Es una herramienta clave de la Mercadotecnia Social en la Salud, por lo que permite transmitir la información de salud de manera clara, vera y oportuna, con sustento en evidencia científica, por lo que busca la sensibilización de la opinión pública, fomentar la participación comunitaria y promover estilos de vida saludables (Secretaría de Salud, 2010, p. 19).

- Comunicación para la prevención de riesgos psicosociales: En cuestión de prevención de riesgos, la estrategia de comunicación es una "herramienta imprescindible para la mejora de la seguridad y salud, ya que permite el intercambio de información necesaria para el cumplimiento del plan de prevención de las empresas, además de impulsar el intercambio de inquietudes y soluciones en materia preventiva" (ISO 2015, p. 8). Una eficaz comunicación permite realizar una prevención más efectiva ya que genera un gran alcance a nivel de sociedad.

\section{Investigación Cualitativa: Entrevistas a profundidad en línea.}

Las entrevistas y los grupos de enfoque en el transcurso del tiempo se han llevado de forma presencial en establecimientos por mutuo acuerdo de los participantes, pero actualmente con el aporte de la tecnología, se ha vuelto más eficiente y accesible obtener las diferentes opiniones necesarias para el complemento de una investigación. McDaniel \& Gates (2016) mencionan que" la entrevista a profundidad en línea suele ser el único medio para llegar a personas como médicos o ejecutivos con múltiples ocupaciones" (p. 147). 


\section{Investigación Cuantitativa: Encuesta en línea.}

La encuesta es uno de los métodos de recolección de información más común a lo largo del tiempo las cuales ha aportado con la toma de decisiones y ha sido fundamental para las investigaciones, gracias a los avances tecnológicos y a las diferentes empresas, se ha logrado llegar a un público con mayor eficacia. McDaniel \& Gates (2016) también menciona: "el número de usuarios de internet sigue incrementando. A medida que el número de usuarios crece a escala mundial y las características de los usuarios en internet tienen tendencia a igualarse" (p. 150). Acorde a lo mencionado por el autor, elaborar y realizar las investigaciones en línea favorecen en cuanto mitigación de tiempo y mayor alcance, además de que gracias a las empresas que proporcionan herramientas como Google brinda la oportunidad de realizar entrevistas y encuestas a través de su plataforma para adquirir información sobre las investigaciones que se realicen.

\section{Bienestar social laboral}

El bienestar es todo lo que se necesita para vivir en un estado óptimo, donde persista un excelente ambiente y se resguarde tanto la salud física como la mental. Por ende, es primordial que en todo ámbito permanezca y se sienta bien, incluyendo en el ámbito laboral. El Bienestar social laboral se define como el estado deseado por un grupo de funcionarios que laboran en la organización, y se logra a través de planes, programas y proyectos, estructurados como solución a las necesidades del empleado que influyen como elemento importante dentro de la empresa (Aguilar, Cruz, \& Jiménez, 2017, p. 23). Orientado a los intereses de los derechos y/o intereses sociales de los trabajadores, además lo que se requiere es complementar y apoyar las políticas generales en este caso de las PYMES, para mejorar sus condicione y así ofrecer al desarrollo del trabajador, la mejora de su nivel de vida y el de su familia.

\section{METODOLOGÍA}

\section{Diseño de investigación}

La metodología de investigación para el presente proyecto fue el método deductivo e inductivo. Método Deductivo por la proporción de información lógica y metódica, que se relaciona con el enfoque cuantitativo, basado en el tipo de investigación descriptiva, con la implementación de encuestas digitales dirigidas a empleadores/representantes legales o el personal administrativo encargado del talento humano de la empresa quienes son encargados las decisiones que se toman en las PYMES, usando como instrumento el cuestionario con preguntas de opción múltiple, dicotómicas y escala de Likert elaboradas por los investigadores del presente proyecto, permitió la obtención de resultados en primera instancia fue conocer el número de PYMES que aplican los programas de riesgos psicosociales en sus empresas, el impacto de los riesgos psicosociales en las PYMES y comprobar el desconocimiento sobre los protocolos en seguridad y salud ocupacional enfocado en los riesgos psicosociales de parte de los dueños o en el personal designado de tomar decisiones. 
Método Inductivo el cual permitió recolectar información desde lo particular a lo general, relacionado con el enfoque cualitativo, y el tipo de investigación exploratoria, el cual permitió establecer una mayor proximidad a la problemática que antecede, con el desarrollo de entrevistas a profundidad estructurada que ayudaron a la presente investigación a obtener información de los riesgos psicosociales que impactan en las PYMES como validación y certificación de información que se recolecta. Además, se realizó un grupo de enfoque con 10 representantes de los trabajadores que se encuentran inmersos en la actividad de comercio, entre 25 a 55 años que cuentan con experiencia laboral, para conocer los factores de riesgos psicosociales que inciden en su comportamiento para el desarrollo de sus actividades. Como instrumento para la entrevista a expertos se utilizó una guía de preguntasestructuradas y para el grupo de enfoque a los representantes de los trabajadores, se usó una guía de preguntas adaptadas de la encuesta a los dueños de las PYMES, con la finalidad de que se validen y comparen proporcionalmente los datos cuantitativos extraídos de la encuesta. El enfoque de investigación fue mixto, por lo que se aplicó ambos enfoques cuantitativo y cualitativo, esto permitió que al combinar ambas concepciones se obtengan resultados que se aproximen a la realidad del problema.

Una vez analizada la base de datos adquirida desde el portal del Servicio de Rentas Internas SRI (2020), se determinó que Guayaquil cuenta con 68.229 PYMES, en la Parroquia Tarqui con 33.851 PYMES, sin embargo por motivos de investigación la población para el presente estudio se sitúa en las 331 PYMES de Guayaquil con estado contribuyente activo y establecimientos abiertos, obligados y no obligados a llevar contabilidad de la Parroquia Tarqui pertenecientes al subgrupo de Venta al por Mayor de Aparatos Domésticos y de uso Domésticos y Venta al por menor de electrodomésticos en establecimientos especializados, los cuales se representan en la Tabla 1, donde se muestran los criterios que a consideración de los investigadores son necesarios para cumplir con la investigación.

\section{Tabla 1}

Minería de datos del SRI

\begin{tabular}{|c|c|c|c|c|}
\hline Población de PYMES & Guayas & Guayaquil & Tarqui & $\begin{array}{l}\text { Subgrupo } \\
\text { (Población) }\end{array}$ \\
\hline $\begin{array}{c}\text { Empresas Actividad De } \\
\text { Comercio - Grupo G. }\end{array}$ & 92.330 & 68.229 & 33.851 & 331 \\
\hline Total & & & & 331 \\
\hline
\end{tabular}

Nota: La información presentada fue realizada con la base de datos obtenida de la página del SRI en el cual presentan datos actualizados de las PYMES registradas en la ciudad de Guayaquil.

Fuente: (SRI, 2020)

\section{Muestra}

En el presente estudio de enfoque Mixto se considera la muestra con relación idéntica. Para el estudio se elige un muestreo probabilístico: muestreo aleatorio simple razonable para el tipo de investigación descriptiva aplicada en encuestas para conocer aproximadamente el número de empleadores/representantes legales y administrativos en $\mathrm{RRHH}$ que aplican programas de riesgos psicosociales en las empresas, corroborando también el conocimiento que tienen acerca de la 
prevención de riesgos psicosociales y de la misma muestra se extrae un porción donde se incluyen a los representantes de los trabajadores de ciertas PYMES para la aplicación de muestreo no probabilístico: por conveniencia para el tipo de investigación exploratoria; y para los expertos en el área que se determine la incidencia de los factores de riesgos psicosociales en PYMES relacionado con los trabajadores y empleadores/representantes legales.

Para la obtención del tamaño de la muestra se estableció conociendo la población objetiva, por lo que se aplica la fórmula para cálculo finito que se detalla a continuación.

Factor de la muestra:

N: Tamaño de la Población. (331)

Z: Parámetro estadístico que depende del Nivel de Confianza (NC) representado en $95 \%=1,96$.

e: Error de estimación máximo aceptado representado en $5 \%$.

p: Probabilidad de que ocurra el evento estudiado (éxito) en $50 \%$.

q (1-p): Probabilidad de que no ocurra el evento estudiado en $50 \%$.

$\mathrm{N}$ : Tamaño de muestra buscado. (178)

$$
\begin{aligned}
& n=\frac{N * Z^{2} * p * q}{e^{2} *(N-1)+Z^{2} * p * q} \\
& n=\frac{331 * 1,96^{2} * 0,5 * 0,5}{0,05^{2} *(331-1)+1.96^{2} * 0,5 * 0,5} \\
& n=\frac{317,8924}{0,825+0,9604} \\
& n=\frac{317,8924}{1,7854} \\
& n=178
\end{aligned}
$$

\section{Distribución de la muestra}

En la Tabla 2, se representa la distribución de la muestra la cual sirve para identificar con claridad la aplicación de instrumentos de recolección de datos.

\section{Tabla 2}

Distribución de la muestra

\begin{tabular}{cccc}
\hline $\begin{array}{c}\text { Técnica de } \\
\text { Investigación }\end{array}$ & Enfoque & Muestra & Involucrados \\
\hline Encuesta & Cuantitativo & 178 & Empleadores/ representantes legales y administrativos de \\
& & & RHH \\
Entrevista & Cualitativo & 2 & Expertos en Seguridad y Salud Ocupacional \\
Grupo de & Cualitativo & 10 & Representantes de los trabajadores \\
Enfoque & & 16 & \\
\hline Total & &
\end{tabular}




\section{RESULTADOS}

A continuación, se detallan los resultados de la encuesta, entrevista y grupo de enfoque realizada en la presente investigación:

\section{Encuesta}

\section{P.1 ¿Realiza usted el Programa de Riesgos Psicosociales que deben cumplir obligatoriamente las empresas?}

Tabla 3

Cumplimiento de los programas de riesgos psicosociales

\begin{tabular}{ccc}
\hline Clases & Frecuencia & $\mathbf{f} \%$ \\
\hline a) Sí & 74 & $41,63 \%$ \\
\hline b) No & 104 & $58,37 \%$ \\
\hline Total & 178 & $100 \%$ \\
\hline
\end{tabular}

\section{Figura 1}

Cumplimiento de los programas de riesgos psicosociales

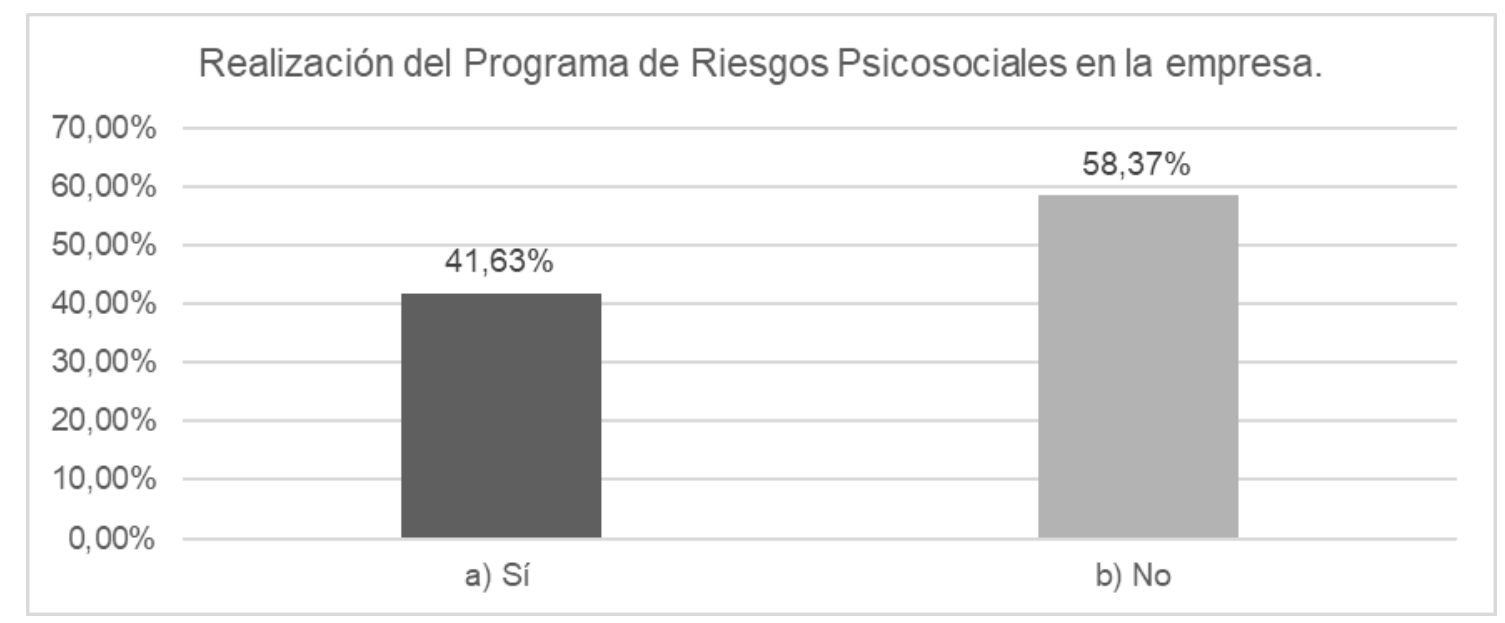

\section{Análisis}

De los encuestados un 58,37\% respondió que no cumple con los Programas de Riesgos psicosociales en la empresa, mientras que un $41,63 \%$ indicó sí cumplir, y a pesar de no haber mucha diferencia en porcentajes, es necesario fomentar que las empresas deben realizar los programas de riesgos psicosociales, el cual favorecerá la cultura organizacional y aportará a una de las obligaciones que debe cumplir el empleador y toda empresa que posea más de 10 trabajadores. De los encuestados que indicaron no realizar programas de riesgos psicosociales, un 93,75\% manifestó que no lo realiza por desconocimiento, sin embargo, un $6,25 \%$ no lo considera necesario, por lo que se debe educar a los 
empleadores qué, por qué y para qué es importante implementar los programas de prevención de riesgos psicosociales dentro de la empresa.

P.2 ¿Participaría en una campaña que le ayude a prevenir los riesgos psicosociales en la empresa?

Tabla 4

Participación en campaña de promoción

\begin{tabular}{ccc}
\hline Clases & Frecuencia & $\mathbf{f} \%$ \\
\hline a) Sí & 145 & $81,71 \%$ \\
\hline b) No & 33 & $18,29 \%$ \\
\hline Total & 178 & $100 \%$ \\
\hline
\end{tabular}

\section{Figura 2}

Participación en campaña de promoción

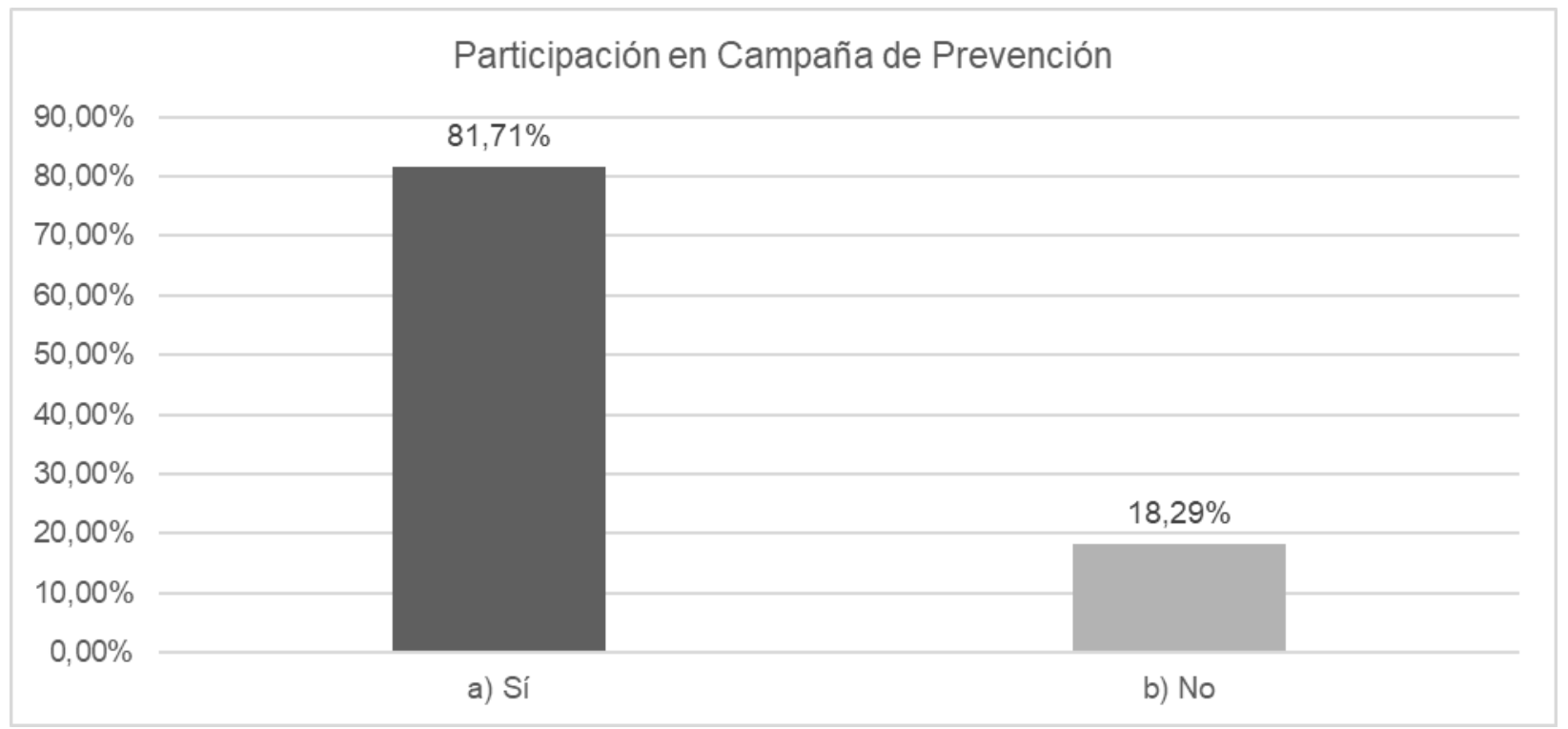

\section{Análisis}

De los encuestados un $81,71 \%$ respondió que sí participarían en una campaña para prevención de riesgos psicosociales, mientras que un $18,29 \%$ indicó que no lo haría. El tiempo es un factor esencial y predominante para el desarrollo de una campaña, y como se puede visualizar los encuestados que indicaron que no participarán en un $70,21 \%$ coincidieron que es debido a la falta de tiempo, seguido de un $19,15 \%$ por recursos económicos no disponibles y un $10,64 \%$ indicó que no cuentan con personal especializado para el desarrollo de la misma, por lo que se debería plantear una Campaña de Prevención de riesgos psicosociales donde se destaque el valor que tienen los programas de riesgos 
psicosociales, que no sea tan costosa y cumpla con las necesidades que tiene el empleador, sin que su ejecución intervenga ampliamente en los recursos disponibles de la empresa.

\section{P.3 ¿Qué medios de comunicación considera idóneos para recibir información sobre la prevención de riesgos psicosociales?}

\section{Tabla 5}

Medios idóneos para recibir información sobre prevención de riesgos psicosociales

\begin{tabular}{lcc}
\multicolumn{1}{c}{ Clases } & Frecuencia & f\% \\
\hline a) Correo electrónico & 46 & $26,07 \%$ \\
\hline b) Grupo de WhatsApp & 44 & $24,51 \%$ \\
\hline c) Canal de Telegram & 16 & $8,95 \%$ \\
\hline d) E-Learning (Plataforma Moodle) & 17 & $9,73 \%$ \\
\hline $\begin{array}{l}\text { e) Redes Sociales (Cuentas de Facebook, } \\
\text { Instagram, Twitter) }\end{array} \quad 55$ & $30,74 \%$ \\
\hline \multicolumn{1}{c}{ Total } & 178 & $100 \%$ \\
\hline
\end{tabular}

\section{Figura 3}

Medios idóneos para recibir información sobre prevención de riesgos psicosociales

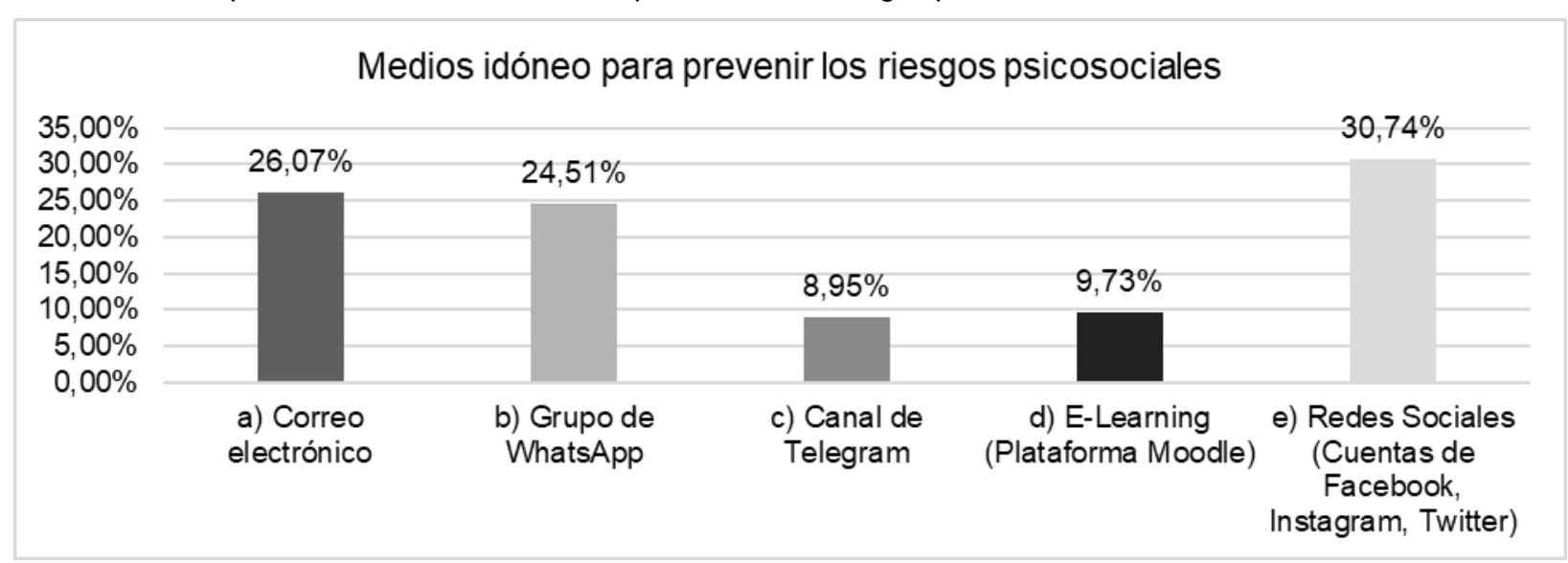

\section{Análisis}

Los medios de comunicación que se consideran idóneos por los encuestados, un 30,74\% concordó que prefiere las redes sociales las cuales abarcan las cuentas de Facebook, Instagram y Twitter, luego están los canales directos como el correo electrónico con 26,07\% y grupo de WhatsApp con $24,51 \%$, consideradas como opciones de preferencia para los encuestados a que su uso sea con la finalidad de recibir información sobre la prevención de riesgos psicosociales.

\section{Entrevista}

E1: Mg. Richard Valenzuela // E2: Mg. Sally Ruales de Haz 
Según los entrevistados mencionan que en Ecuador los riesgos psicosociales que tienen mayor incidencia son el Mobbing conocido como el acoso laboral y el síndrome de Burnout que es un nivel de estrés extremadamente crónico producido cuando la persona siente un nivel de agotamiento extremo pero sin embargo sigue siendo presionando y ejerciendo las labores inclinándose a una serie de factores negativos que no le permite desenvolverse en un ambiente laboral favorable, además, si llegan a tener mayor repercusión podría provocar hasta el suicidio en quienes lo padezcan, generando así otro problema por atender de parte de la empresa.

Los entrevistados estuvieron de acuerdo en que se debe implementar una campaña de concientización sobre los riesgos psicosociales a los representantes legales y al personal administrativo de RRHH que está a cargo de los trabajadores en las PYMES, debido a que a pesar de que algunos están conscientes del problema que existe en cuanto a la falta de prevención de riesgos psicosociales y/o cualquier otro nivel, no están haciendo nada por mitigarlo por lo que sugieren se realice campañas por las diferentes vías como: WhatsApp, Telegram que generalmente no son solo para dialogar si no que ayudan a la creación de repositorios de información tal como una biblioteca virtual para que los representantes legales utilicen ese medio y adquieran información al instante.

\section{Grupo de Enfoque}

En la presente intervención se contó con la participación de 10 representantes de los trabajadores, cinco hombres y cinco mujeres, con varios años de experiencia laboral, quienes en su mayoría cumplen con las 40 horas laborales, a pesar de que hubo algunos que indicaron trabajar 20 horas y más de 40 horas semanales. En los resultados de la evaluación de las condiciones laborales que tienen los trabajadores en las PYMES se identificó que acorde a los factores existen diferentes factores que los altos mandos no están considerando importantes en el desarrollo de las actividades de sus trabajadores.

En lo que respecta al factor carga y ritmo de trabajo. - acorde a la información recabada en el grupo focal, los participantes muestran cierto malestar referente a su jornada laboral debido a que en algunos casos no suelen reconocer sus horas adicionales o horas extras, adicionalmente indican que deben laborar fines de semana a pesar de que en su contrato no estipulaba aquello. También comentaban que tiene un exceso de trabajo ya que sus horas de trabajo no son suficientes para todo lo que les asignan, llevando parte de su trabajo a la casa lo cual les merma el tiempo para realizar actividades personales y familiares. Con esto se puede establecer un alto riesgo psicosocial en estas empresas debido al poco interés que tienen al bienestar de sus trabajadores y de su desempeño dentro de la organización.

Relacionado al factor de organización en el trabajo, lo indicado por los participantes sobre si la comunicación dentro de sus empresas es correcta, varios mencionaron el malestar que les ocasiona la inexistencia de una buena comunicación entre sus jefes inmediatos o con otras áreas dentro de la organización, de igual manera indican que la empresa no suele comunicar claramente puntos 
importantes para ellos y sus labores. Muchas veces entre las mismas jefaturas hay una gran falta de comunicación, lo que complica el trabajo que deben realizar los colaboradores.

Los participantes fueron muy expresivos referente al factor de soporte y apoyo, al consultar acerca de si la empresa sancionaría algún tipo de acoso o discriminación, la mayoría indicó que sí se cumple este aspecto dentro de la organización o al menos ellos considerarían que así lo harían. Por el contrario, al consultar sobre si se realizan capacitaciones sobre temas relacionados a la prevención de riesgos psicosociales en su totalidad indicaron que sobre estos temas no hay casi ningún tipo de capacitación al respecto, algunos mencionaron que brevemente al ingresar a la organización, pero muy superficialmente, esto nos da una clara idea sobre el poco interés que hay en las empresas acerca de informar a su personal sobre riesgos a los cuales podrían estar expuestos.

\section{CONCLUSIONES}

Una vez analizado los instrumentos utilizados en la presente investigación como resultado se obtuvo que los riesgos psicosociales impactan de forma negativa en las PYMES debido a que existe una deficiente gestión en cuanto a organización, comunicación y prevención de riesgos psicosociales en los que se perjudican desde los directivos de altos mandos y medios hasta a los trabajadores y la sociedad en general, ya que todos los aspectos relacionados en salud mental tiene su influencia por factores internos y externos de la empresa. Según los factores que fueron evaluados, los riesgos psicosociales que deben ser atendidos principalmente son el estrés, burnout y mobbing, ya que tienen una mayor repercusión dentro de las empresas.

Para lograr ese cambio que requieren las PYMES, de la investigación se extrajo que los empleadores, representantes legales y administrativos de Talento Humano se sensibilicen con una campaña social para la prevención de riesgos psicosociales a través de la educación, concientización y fomento de la cultura de prevención que permita generar ese conocimiento o esa actualización de cómo gestionar apropiadamente los riesgos psicosociales dentro de la empresa usando medios de comunicación accesibles y asequibles como redes sociales (Facebook, Instagram y Twitter), aplicaciones para reuniones (Google Meet \& Zoom) y de mensajería (WhatsApp) con la finalidad de proporcionar información relevante al grupo en mención.

La investigación demostró que sí es necesario proponer estrategias de marketing social con tono educativo, por lo que brindan la opción de ser adaptadas a la situación actual, en las cuales se integró las estrategias de marketing general: estrategia básica de desarrollo, estrategia de cambio social usando la herramienta de educación para lograr ese modificación en el comportamiento y se torne voluntario, además con los medios de comunicación digitales que se planteó, se espera un mayor alcance de beneficiarios. 


\section{REFERENCIAS}

Aguilar, V., Cruz, V. H., \& Jiménez, D. (2017). Bienestar social laboral desde la perspectiva de calidad de vida a partir de la producción escrita y la percepción de docentes especialistas: periodo 1995 2005 en Bogotá D.C. Estado del Arte. Ciencia Unisalle, 1-258. Obtenido de https://ciencia.lasalle.edu.co/trabajo_social/64/?utm_source=ciencia.lasalle.edu.co\%2Ftrabajo_s ocial\%2F64\&utm_medium=PDF\&utm_campaign=PDFCoverPages

ISO 2015. (15, Septiembre 2015). Norma Internacional ISO 9001 - 2015 Sistemas de gestión de la calidad. Retrieved from Traducción Oficial:

http://www.itvalledelguadiana.edu.mx/ftp/Normas\%20ISO/ISO\%2090012015\%20Sistemas\%20de\%20Gesti\%C3\%B3n\%20de\%20la\%20Calidad.pdf

Kotler, P., \& Armstrong, G. (2013). Fundamentos de Marketing (11ava ed.). (G. D. Chávez, Ed.) México: Pearson Educación de México. Recuperado el 28 de enero de 2021

McDaniel, C., \& Gates, R. (2016). Investigación de Mercados (10ma ed.). México, D. F.: México: Cengage Learning.

Ministerio de Salud Pública del Ecuador. (2019). Política Nacional de Salud en el Trabajo 2019 -2025. Obtenido de https://www.salud.gob.ec/wp-content/uploads/2019/10/MANUAL-DE-POLITICASfinal.pdf

Ministerio de Salud Pública del Ecuador. (2020). Protocolo de Prevención del Riesgo Psicosocial en intervinientes Operativos y Administrativos. Obtenido de https://www.salud.gob.ec/wpcontent/uploads/2020/08/protocolo-de-prevenci\%C3\%93n-de-riesgos-psicosoc-mtt2-_2020_2.pdf

Robalino Zumba, J. A. (2020, Julio 28). EVALUACIÓN DE LOS FACTORES PSICOSOCIALES ANTE EL COVID19 EN EL PERSONAL DE UN SUPERMERCADO DE LA CIUDAD DE QUITOECUADOR. Retrieved from https://repositorio.uisek.edu.ec/bitstream/123456789/3861/2/TesisAlejandro\%20Robalino_compressed.pdf

Secretaría de Salud. (Julio de 2010). Manual de Mercadotecnia Social en Salud. Obtenido de https://www.uv.mx/cendhiu/files/2018/02/Manual_Mercadotecnia.pdf

Seguí, G., \& Pérez, H. (Mayo de 2016). Plan de Marketing Social para el proyecto de transferencia de los sistemas de riego estatales a los usuarios - Caso Sistema de Riego Cebadas. Journal of Comunication, 1-42. Obtenido de https://gredos.usal.es/bitstream/handle/10366/142497/Estrategia_de_Marketing_Social_propue st.pdf?sequence $=1$ \&isAllowed $=y$

SGRT. (2020). Reporte de Atenciones Médicas. Obtenido de https://app.powerbi.com/view?r=eyJrljoiYWJhOTImYjYtZmJjOS00Y2ZiLWI4Y2UtNzFiMDZhMjAx ODA4liwidCI6ljZhNmNIOGVkLTBIMGYtNDY4YS05Yzg1LWU3Y2U0ZjIXZjRmMiJ9\&pageName= ReportSection584b3d0d5ae8994c488c

SRI. (2020). Obtenido de https://www.sri.gob.ec/catastros 
Tandalla, S. (2018, Junio). CULTURA PREVENTIVA: ESTRATEGIAS DE CAMBIO CULTURAL DE OBLIGATORIEDAD A CONCIENCIACIÓN DE PREVENCIÓN DE RIESGOS LABORALES PARA GENERAR EXCELENCIA, SALUD EMPRESARIAL Y SOSTENIBILIDAD. Catalunya: Escuela Politécnica Superior d'Edificació de Barcelona. Retrieved from https://upcommons.upc.edu/bitstream/handle/2117/133780/Mem\%c3\%b2ria_TandallaSandra\%2 0Marcela.pdf? sequence $=1$ \&isAllowed $=y$ 